welches besagt, daß je zwei Massenpunkte $M$ und $m$ sich gegenseitig mit Kräften anziehen, deren Größe $P$ dem Produkte $M m$ direkt und dem Quadrat ihres Abstandes $r$ umgekehrt proportional ist. Inbezug auf die Planetenbewegung stimmen die beiden Annahmen (71) tnd (72) überein, wenn man von der verschwindend kleinen Bewegung absieht, die der Sonne von dem Planeten erteilt wird. Denn das Produkt $x M$ aus der Gravitationskonstanten $*$ und der Sonnenmasse $M$ kann durch die Bezeichnung $"$ ersetzt werden.

9. Schlufbemerkung. In den vorstehenden Betrachtangen ist von den Hauptsätzen der Mechanik nur ein einziger zar Anwendung gekommen: der Satz von der geometrischen Summierung der Bewegungsgrößen $m v$ und der Kraftantriebe Pdt. "Die Entdeckung dieses Satzes könnte seiner Einfachheit wegen als nuerheblich orsoheinen. Man muß aber bedenken, daß vorher die Begriffe: Masse. Kraft, Bewegungsgröße und Kraftantrieb gebildet werden mußten. Es ist das nnvergängliche Verdienst New tons, hierdurch die Grundlage der heutigen Dynamik geachaffen zu haben. Vor Newtong Zeit waren diese Begriffe kaum vorhanden oder doch sehr unklar und versohwommen. Deshalb konnte Kepler seine Gesetze und Hooke seine Hypothese nicht weiter verfolgen. Mit Hilfe der grundlegenden Begriffe ergab sich das Gesetz der ebenen Bewegung eines materiellen Punktes freilich fast von selbst.

\title{
Über die Spannungserhöhung durch kreisförmige Löcher in einem gezogenen Bleche.
}

\author{
Von TH. PóSCHL in Prag.
}

$\mathrm{D}$ ie Frage, in welchem Maße die Spannung in einem Zug oder Druok unterworfenen Körper durch Anbringen von Durchlochungen beeinflußt wird, ist in sehr vielen Füllon von praklischer Bedeutung. Sie läßt sich, wenigstens bei einfachen Annahmen über die Gestalt und Anordnung der Löoher, mit den Hilfsmitteln der Elastizitätstheorie streng erledigen. In einer demnächst erscheinenden Mitteilung ${ }^{1}$ ) habe ich den Einflub eines elliptischen Loches in einer in irgend einer Richtung oder allseits gleichförmig gespannten Soheibe untersucht. Wie bei allen derartigen Problemen kam es vor allem naturgemäb darauf an, angemessene Koordinaten zu verwenden, als welche sich die elliptischen Koordinaten von selbst darboten. Die zwoiten kovarianten Ableitungen der zugehörigen Airy schen Funktion stellen dann die Spannungen an jeder Stelle des Bleches dar.

Hat man die Spannungsverteilung zu ermitteln, die durch zwoikreisförmige Löcher in einem gespannten Bleche ${ }^{2}$ ) herporgerufen wird, so wird man als Koordinatensystem das Doppelsystem der Kreise zweier Büschel verwenden, das za zwei reellen und zu zwei imaginären Grundpurkten gehört (bizirkulare oder bipolare Koordinaten). Auch dieses System ist isometrisch (d. h, die Kurvenvierecke sind quadratisch), und dieser Umstand bringt, wie anch im eben erwhhnten elliptischen Problem, eine wesentliche Vereinfachung in der Rechnung mit sich, wenn es auch - da es sich doch um die Gleichung $\Delta\lrcorner \psi=0$ handelt - unmöglich ist, das Hilfsmittel der konformen Abbildnng unmittelbar zu verwerten.

In der vorliegenden Note gebe ich die Entwicklung zunächst für den Fall, daß die in zwei Kreisen durohlochte Seheibe in radialer Richtung allseits gleichmäßig gespannt ist; es zeigt sich, wie nicht anders zu erwarten, daß der gröbte Wert der Tangentialspannung an den inneren, zueinander nächstliegenden Punkten der beiden Kreise auftritt, und daß dieser größte Wert am so größer ist, je größer die Durchmesser der beiden Kreise sind nnd je näher die beiden Kreise einander liegen. Im folgenden sind die hierfür geltenden Formeln auch zahlenmäßig ausgewertet. Die erhaltenen Ergebnisso liefern einen Beitrag für die Beurteilung der Größe der Spannangserhöhung, die durch mehrere Löcher entstehen, z. B. durch Nietlöcher in einem Bleche, wenn auch bierbei

J) Math. Zeitgehr. 11, 1921, S. 1 bis 7 .

5) Es basdelt sich stets om das ebene Problem, das bekauntlich In folgenden beiden Fallen vorliegt: a) befm unenduich laugen Zyliıder, der grleishıäßig seiner gauzeı Lange nach beansprucht wird, b) bei der unendlich dunnon scheilue. 
teilweise andere Verhaltnisse herrsohen. Ein.weiteres, sich an die vorliegende Note anschließendes Problem wäre die Untersuchung der Spannungsverhältnisse für die unendliche Kreisreihe (Kreisgitter) $\mathrm{u}$. dergl. $\mathrm{m}$.

Die Ermittlung der Spannungsverteilung für den Fall, daß das Blech nur nach oiner Richtung gespannt ist, habe ich bisher nicht vollständig erledigen können, da die Erftillung der Bedingungen im Unendlichen hierbei Schwierigkeiten bereitet.

1. Grundgleichungen. Von Cartesischen Koordinaten $x, y$ in der Ebene ausgehend, werde ein System von orthogonalen krummlinigen Koordinaten $\xi$, $\eta$ eingeführt mittels dèr Funktion

oder

$$
\xi+i \eta=1 / 2 \log \frac{i c \ldots z}{i c+z}
$$

daraus folgt zurächst

$$
z=x+i y=\text { c. } \operatorname{tg} 1 / 2(\xi+i \eta)
$$

also

und ebenso

$$
\operatorname{tg} \xi=\operatorname{tg} 1 / 2(\xi+i \eta+\xi-i \eta)=\begin{gathered}
2 c x \\
c^{2}-x^{2}-y^{2}
\end{gathered}
$$

Außerdem folgt aus Gl. (1)

also

$$
x^{2}+y^{2}+2 c x \operatorname{ctg} \xi-c^{2}=0 \text {. }
$$

$$
x^{2}+y^{2}-2 c y\left(5 \operatorname{tg} \eta+r^{2}=0\right.
$$

$$
x+i y=\frac{\sin ^{1} / 2\left(\xi+i \eta_{1}\right) \cos 1 / 2(\xi-i \eta)}{\cos 1 / 2(\xi+i \eta) \cos ^{1 / 2}(\xi-i \eta)}=c \frac{\sin \xi+i \sin \eta}{(5) \mid \eta+\cos \xi},
$$

$$
\left.\begin{array}{cc}
x=c & \sin \xi \\
& \operatorname{sof} \eta+\cos \xi \\
\operatorname{Sin} y \\
=c & \operatorname{soj} \eta+\cos \xi
\end{array}\right)
$$

Die Gleichung (2) stellt eine Schar von Kreisen dar, deren Mittelpunkte $C$ auf der $x$-Achse in einer Entfernung $-c$ otg $\xi$ von 0 liegen, Abb. 1, und deren Halbmesser gleich $c$ cosec $\xi$ sind. Diese Kreise bilden ein Kreisbüschel, dessen Grundpunkte $A$ und $B$ auf der $y$-Aohse in den Abständen $\pm c$ von 0 liegen.

Dabei ist $\Varangle \xi=\Varangle O C A$; daher stellt $\xi=0$ den Teil $A B$ der $y$-Achse dar; wenn $0<\xi<\pi$, so besteht die Kurve $\xi=$ konst. aus dem Stück eines.Kreisbogens zwischen $A$ und $B$, das auf der positiven Seite der $x$ Achse liegt; für $\xi=\pi$ wird die Kurve die ganze $\boldsymbol{y}$-Achse außerhalb des Teiles $\boldsymbol{A} B$. Wenn $-\pi<\xi<0$, so erhält man jene Kreisbogenstücke, die auf der negativen Seite der $x$-Achse liegen.

Die Gleichung (3) stellt dagegen das System der Orthogonalkreise zu diesen Kreisen dar; es besteht aus zwei getrennten Scharen, deren Mittelpunkte auf der $\boldsymbol{y}$-Achse in Entfernungen $\pm c .5 \operatorname{tg} \eta$ von 0 liegen, und deren Halbmesser gleich $c$ Sujec $\eta$ sind. Diese Kreise schneiden die $x$-Achse nicht. Wenn $\eta=\infty$ ist, so erhält man den Punkt $A$; für $\infty<\eta<0$ stellt

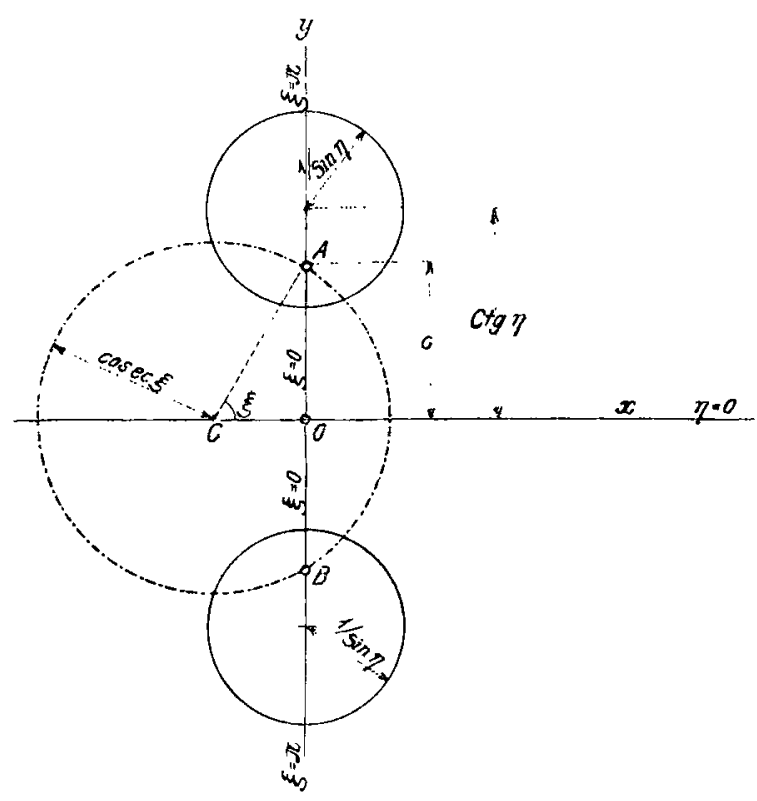

Abb. 1 $\eta=\eta_{0}=$ konst. einen Kreis dar, der $A$ umgibt; für $\eta=0$ erhält man die $x$-Achse. Für $-\infty<\eta<0$ liefert $\eta=$ konst. einen Kreis, der $B$ umgibt, welcher Punkt selbst dem Werte $\eta=-\infty$ entspricht. 
Das Unendliche wird daher durch $\xi=\pi, \eta=0$ dargestellt.

Drïcken wir das Bogenelement durch diese Koordinaten $\xi$ und $\eta$ aus, so folgt

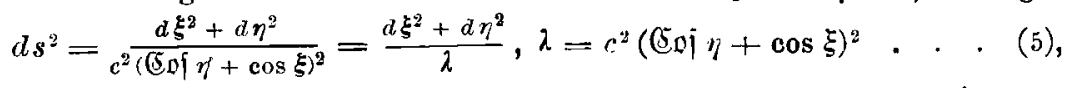

und wenn wir für die Laplaceschen Differentialausdrücke die Bezeichnungen $\Delta \psi=\psi_{x x}$ $+\psi_{\varphi \varphi}$ bzw. $\Delta^{*} \psi=\psi_{\xi \xi}+\psi_{\eta \eta}$ verwenden, so ist

$$
\Delta \psi=\lambda \Delta^{*} \psi \text {. . . . . . . . . . . . . (6). }
$$

Wir betrachten nun ein unendlich langes Blech, das mit zwei kreisförmigen Löchern versehen ist, die durch $\eta= \pm \eta_{0}=$ konet. gekennzeichnet sind, wobei $0<\left|\eta_{0}\right|<\infty$, und an deren Rändern keine äußeren Kräfte angreifen mögen.

Nach dem bekannten Vorgange ${ }^{1}$ ) hat man zur Lösung derartiger Aufgaben eine über die Ebene ausgebreitete Fanktion, die Airysche Spannangsfunktion auf Grund folgender Forderungen zu bestimmen:

I. Im ganzen Bereiche außerhalb der gegebenen Begrenzungen genügt $\psi$ der »biharmonischen Differentialgleichung"

$$
\Delta \Delta \psi=0 \text {. . . . . . . . . . . . (7), }
$$

die in den für das Doppelkreissystem geltenden Koordinaten $\xi$ und $\eta$ so lautet:

$\left(\operatorname{coj}_{\eta}+\cos \xi\right) \Delta^{*} \Delta^{*} \psi-4\left(\sin \xi \frac{\partial \Delta^{*} \psi}{\partial \xi}-\operatorname{Sin} \eta \frac{\partial \Delta^{*} \psi}{\partial \eta}\right)+4\left((50 i \eta-\cos \xi) \Delta^{*} \psi=0\right.$.

II. Längs des Umfanges der beiden Kreise $\eta_{0}=$ konst. schließt sich die Funktion $\psi$ stetig und mit stetiger Tangente an die über den Kreisen aufgebante äuBere Belastungsfläohe ${ }^{2}$ ) an.

Wenn im Innern dieser Kreise keine Kräfte wirken, so besteht diese äußere Belastungsfläche aus zwei über den Kreisen liegenden Ebenenstiicken, die aus Symmetriegründen nur lïngs der $y$-Achse, und zwar symmetrisch zur $x$-Achse (duchförmig) geneigt sind. Die Neigung dieser Ebenen ist nicht von vornherein gegeben, sondern wird vielmehr durch das Problem selbst bestimmt.

III. Im Unendlichen muß $\psi$ den Bedingungen für gleichförmigen Zug nach irgend einer Richtung bezw. gleichförmigen radialen $\mathrm{Zug}$ genügen ${ }^{3}$ ), mub also von der Form sein:

a) $\psi \rightarrow \frac{p}{2} r^{2}$ bei gleichförmigem, radialem $\mathrm{Zug}$,

b) $\psi \rightarrow \frac{p}{2} x^{*} \gg \quad$ Zug in der Richtung $y$,

c) $\psi \rightarrow \frac{p}{2} y^{2} \gg \quad$ • $\gg, x$,

d) $\psi \rightarrow \frac{p}{2}(x \cos \alpha+y \sin \alpha)^{2}$ bei gleichförmigem $\mathrm{Zug}$ in einer unter dem Winkel $\alpha$ gegen

die $y$-Achse geneigten Richtung.

Trägt man $\psi=\psi(\xi, \eta)$ an jeder Stelle der Ebene als Ordinate auf, so erbält man die Spannungsfläche oder innere Belastungsfläche, für welche die Bedingungen II nnd III unmittelbar einleuchtende geometrische Bedeutung haben.

In der vorliegenden Note beschaftigen wir uns vorerst mit dem Fall IIIa.

2. Spannungsfunktion für gleichförmigen radialen Zug. Aus Symmetriegründen muß die gesuchte Funktion sowohl in $\xi$ wie auch in $\eta$ gerade sein. Die Bedingungen II. und IIIa. fiir die Funktion $\psi$ können wir nunmehr so aussprechen:

I. In der ganzen Ebene außerhalb der beiden Kreise ist $\Delta \Delta \psi=0$.

II. An den Rändern der Kreise ist

$$
\text { fïr } \eta=\eta_{0}, \psi=C y
$$

1) s. F. Klein und K. Wicghardt, Ueber Spannungsflachen und rezlproke Diagramme, Archiv Nath. Plyys. (3 Reibe) 8, 1904, S. 1 und 95.

2) s. P. Funk, Die linearen Differenzengleichungen und ilıre Anwendung in der Theorle der Baukonstruktionen, Berlin 1920.

3) Die Bedingungen lauten genaner so, daß die zweiten Ableitungen von $\%$ sich in linendlicben wie die der rechtsstehenden Ausdrucke von a) bis d) verlalten, praktisch komml dies auf die im $T(x)$ gegebene Fassung binaus 
nnd

$$
\text { fiir } \left.\begin{array}{rl}
\eta & =\eta_{0}, \frac{\partial \psi}{\partial \eta}=C \frac{\partial y}{\partial \eta} \\
& \eta=-\eta_{0}, \frac{\partial \psi}{\partial \eta}=-C_{\frac{\partial y}{\partial \eta}}
\end{array}\right\}
$$

$C$ gibt die Neigang der Ebenen und wird durch das Problem selbst bestimmt; $y$ ist durch Gl. (4) gegeben.

III a. Für $\xi=\pi, \eta=0$ ist

$$
\eta \rightarrow \frac{p}{2} r^{2}
$$

Was die Auswahl der Potentiale und Bipotentiale, aus denen sich die gesuchte Lösung zusammensetzt, anlangt, so wird man es zunächst mit den einfachsten versuohen. Wegen der Gleichung (10) wird man vor allem $r^{2}$ selbst heranziehen, wobei

oder auch

$$
r^{2}=c^{2} \frac{\cos _{0} \eta-\cos \xi}{(50 ; \eta+\cos \xi}
$$

$$
\frac{1+\eta^{2}}{2}=c^{2} \frac{(50 f \eta}{50 f \eta+\cos \xi}, \frac{1-r^{2}}{2}=c^{2} \frac{\cos \xi}{(50) \eta+\cos \xi} \text {. }
$$

Alle noch hinzutretenden Anteile müssen im Unendlichen von niedrigerer Ordnung unendlich werden, und zwar müssen sie, da sie gerade Funktionen sind, regulär sein. Der Umstand fiibrt sofort auf

$$
\eta y=\frac{\eta \operatorname{Sin} \eta}{\operatorname{Soj} \eta+\cos \xi}
$$

In der Tat ist die gesuchte Lösung von der Form

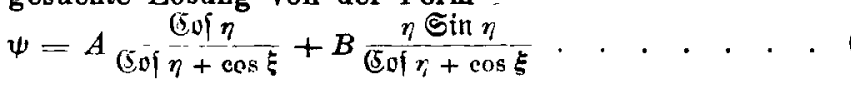

wenn wir mit $A$ und $B$ Konstante bezeichnen. G]. (8) gibt sogleich

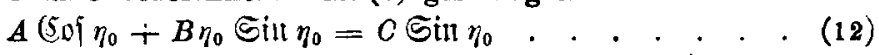

und Gl. (9) gibt nach Ausführung der Differentiation nach $\eta$ in den Ausdrücken (11) und (9)

$$
\left.\begin{array}{rl}
A \text { Sin } \eta_{0}+B\left(\eta_{0}\left(\operatorname{Soj}_{0} \eta_{0}+\operatorname{Sin}_{0}\right)=C \operatorname{Soj} \eta_{0}\right. \\
B\left(\eta_{0}+\operatorname{Soj}_{0} \eta_{0} \operatorname{Sin} \eta_{0}\right)=C
\end{array}\right\}
$$

Die Gleichungen (12) and (13) sind miteinander verträglich; ihre Determinante verschwindet identisch. Es ergibt sich daraus

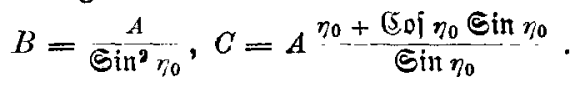

Wenn wir endlich die Spannung pro Längeneinheit im Unendlichen mit $p$ bezeichnen, so folgt aus

fïr $A$ die Aussage

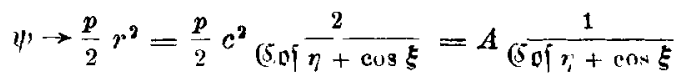

$$
A=p c^{2} \text {. }
$$

Wir erhalten daher die gesuchte Spannungsfunktion in der einfachen Gẹstalt

$$
\psi=p c^{2} \frac{\varsigma_{0} \eta+\frac{1}{\operatorname{Sin}^{2} \eta_{0}} \eta \text { } \operatorname{Sili} \eta}{\varsigma_{0} \mid \eta+\cos \xi}
$$

3. Die Spannungskomponenten. Wie Ich in der eingangs erwăhnten Arbeit auseinandergesetat habe und wie durch sinngemäBe Auflassung des Begriffes Differentialquotient einleuchtet, sind die Spannungskomponenten in den Koordinaten $\xi$, für die wir in der Loveschen Bezeichnung $\xi \xi, \widetilde{\eta \eta}$ and $\widetilde{\xi}$ schreiben, duroh die Produkto der bzgl. zweiten kovarianten Ableitungen von $\psi$ nach den Koordinaten mit $\lambda$ dargestellt. Die Spannung; die uns insbesondere interessiert, ist die Tangentialspannung $\widehat{\xi} \xi$, fïr die sioh folgender Ausdruck ergibt:

$$
\overline{\xi \xi}=\lambda \psi_{i_{i}}-1 / 2 \lambda_{\xi} \psi_{\xi}+1 / 2 \lambda_{\eta} \psi_{\eta}
$$

und für $\lambda$ seinen Wert eingesetzt

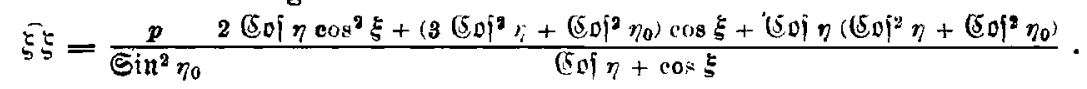


Für den Rand der Kreise $\eta= \pm \eta_{0}$ erhält man den einfachen Ausdruck

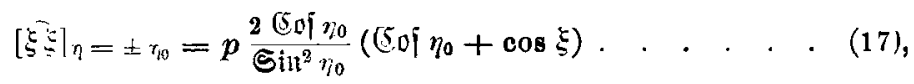

während die beiden anderen Spannungskomponenten $\hat{i \eta}$ und $\overline{\xi \eta}$, die durch analoge Gleichung wie (15) gegeben sind, für $\eta= \pm r_{0}$ identisch 0 ergeben.

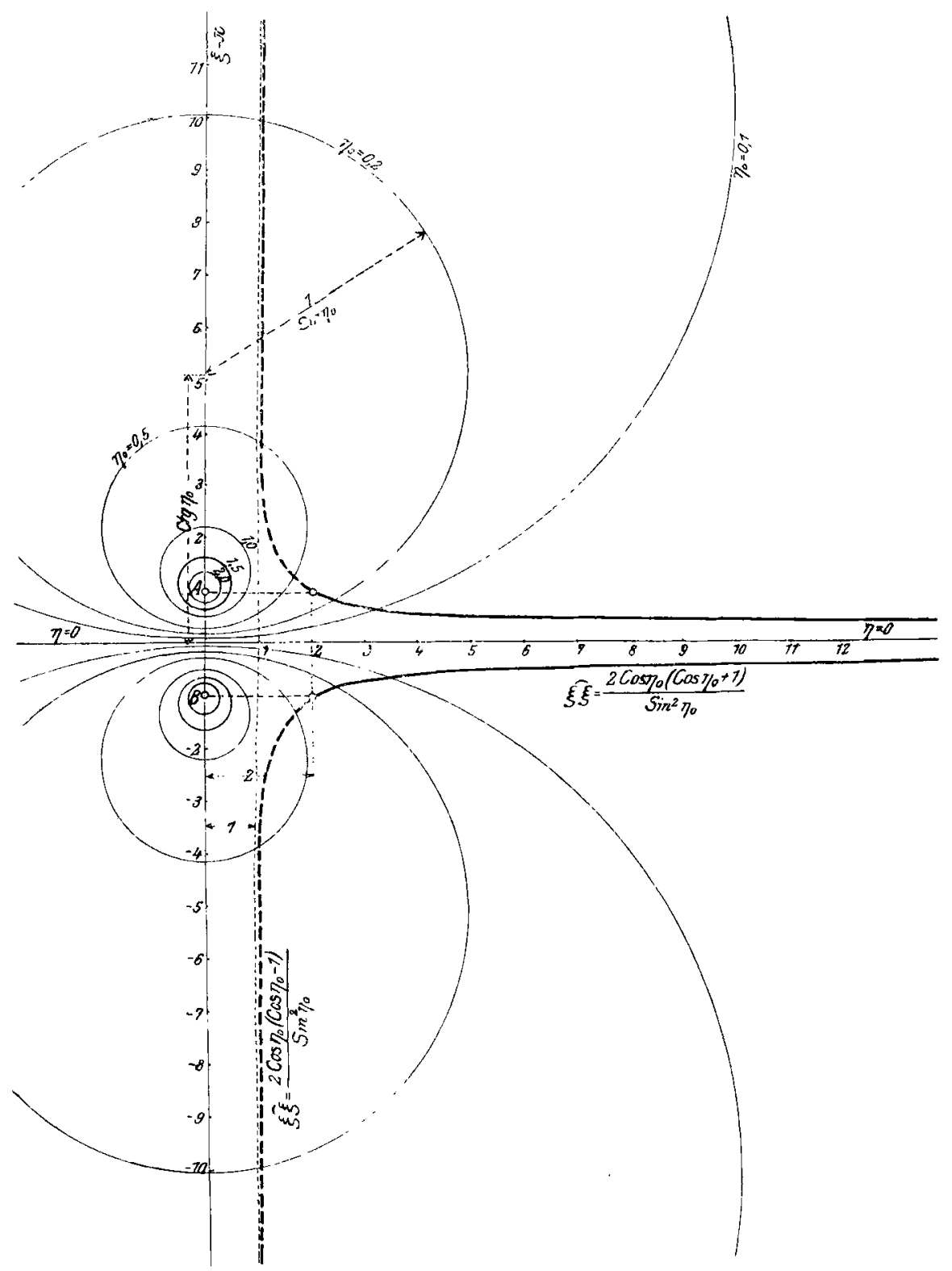

Ab). 2

In Abb. 2 ist zunærchst die Tangentialspannung $\oint_{\zeta}$ an jenen Durchmesserendpunkten, in welchen die Verbindungegerade der Mittelpunkte der Kreise (d. i. die $y$-Acbse) diese schneidet, für alle Kreise des durch den Wert $c=1$ gekennzeichneten Büschels dargestellt; dabei ist auch $p=1$ gesetzt. Die dafür geltenden Formeln sind nach Gl. (1 7 ) 


$$
\begin{aligned}
& \text { innerer Durchmesserendpunkt }(\xi=0): \hat{\xi}=\frac{2 \text { (E) } r_{0}\left(E 0 j \eta_{0}+1\right)}{\operatorname{Sin}^{2} r_{0}} \text {, }
\end{aligned}
$$

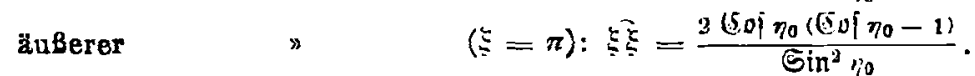

Für andere $p$ sind diese Werte mit $p$ zu multiplizieren. Die Spannungen an den inneren Durchmesserendpunkten stellen sich weit höher heraus wie außen, und zwar um so böher, je größer die Kreise werden, je näher sie also der $x$-Achse kommen, d. $h$. je nohmäler der zwisohen den Kreisen bleibende Quersehnitt wird. Wenn die Kreise auf das Paar der Grundpunkte $A$ und $B$ einschrumpfen $\left(\eta_{0}= \pm \infty\right)$, so nahert sich EE dem Werte 2, und dies ist jener Wert, den die Spannung nuch fïr ein einzelnes Kreisloch

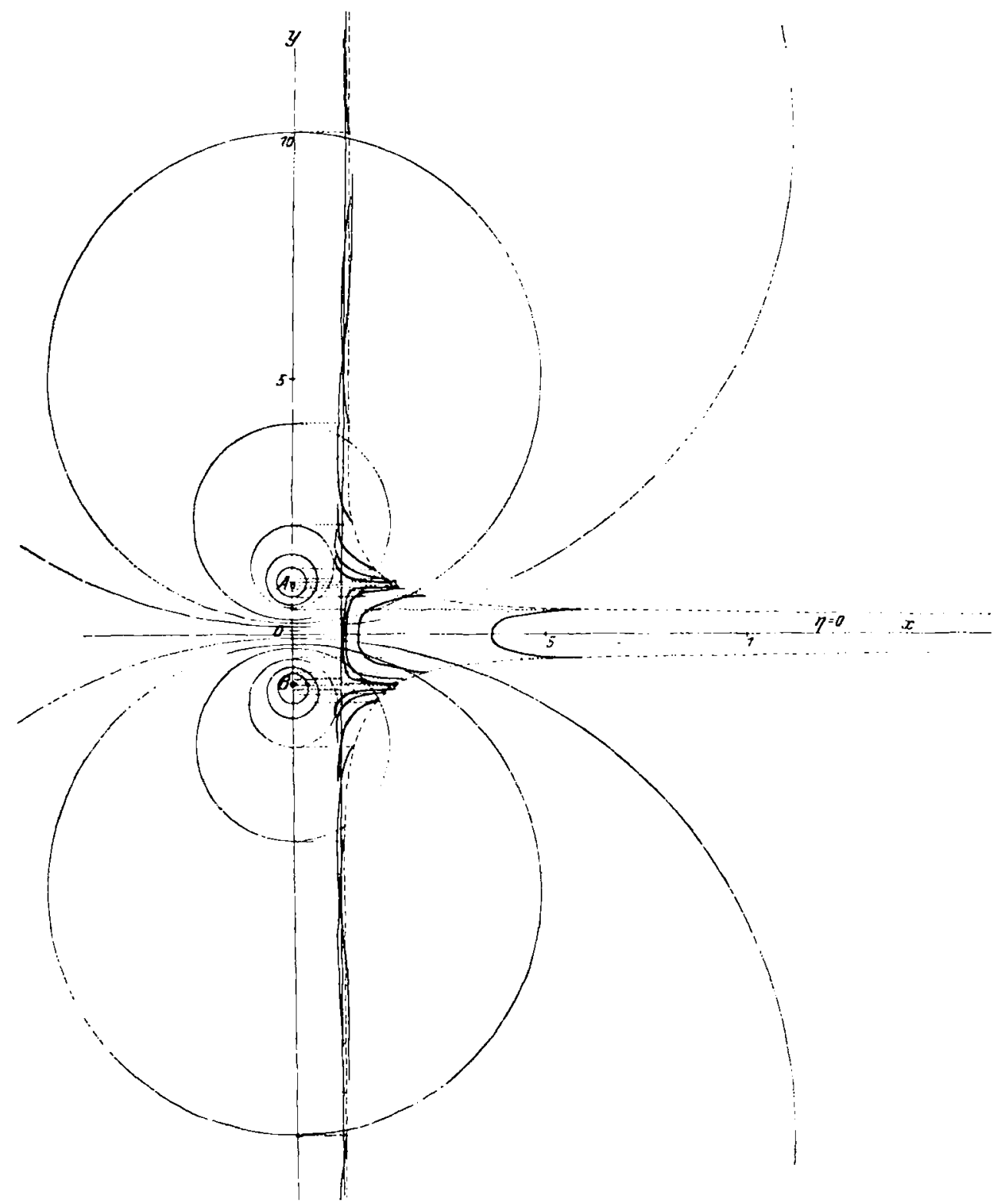

Abb. :3 
(von beliebiger Größe) in einem allseits gleichmäßig gespannten Bleche annimmt ${ }^{1}$ ). Für zwei Löcher (und wahrscheinlich auch für beliebig viele, die sich nicht hăufen) tritt dieser Wert nur für den Fall auf, wo es sich um unendlich kleine Löcher, also um Punktierungen handelt.

Ferner ist in Abb. 3 für eine Anzahl von Kreispaaren der Verlauf der Spannung $\widehat{\xi} \xi$ längs der außerhalb der Kreise verlaufenden Stücke der Verbindungslinie der Mittelpunkte aufgetragen. Die so erhaltenen Kurven erscheinen zwischen den zwei Aesten der in Abb. 2 dargestellten Kurve eingebängt, die hier punktiert eingetragen ist.

Leon und Zidlicky ${ }^{2}$ ) haben für die nach einer Richtung gespannte doppelt darchlochte Scheibe (Kautschnkplatte) Versuche angestellt und auch die Spannungsverteilung $\mathrm{z}$ Kischen den Löchern dargestellt; die hier erhaltenen Ergebnisse stimmen damit qualitativ gut überein. Zahlenmäßig läßt sich ein Vergleich wegen der verschiedenen Belastungen nicht durchführen.

Die numerischen Werte von $\widehat{\xi} \xi$ sind aus folgender Zahlentafel zu ersehen.

a) Innorhalb der Lücher $(\underline{\xi}=0)$

b) auBerbalb der Lücher $(\xi=\pi)$

\begin{tabular}{|c|c|c|c|c|c|c|c|c|c|c|c|c|c|c|}
\hline$r_{i 0}=$ & 0,1 & 0,2 & $0, \mathbf{5}$ & 1.0 & $2, n$ & 3,0 & 4,0 & 0,1 & 0,3 & 0,5 & 1,0 & 2,0 & 3,0 & 4,0 \\
\hline
\end{tabular}

Anwendung der elementaren

Turbinentheorie auf die Berechnung der Windräder.

Von C. PFLEIDERER in Braunschweig. .

$\mathrm{A}^{\mathrm{n}}$

ngesichts der heutigen Notlage unseres Wirtschaltslebens wird der Ausnutzung der in den bewegten Luftmassen enthaltenen Energiemengen besonderes Interesse zugewandt. Tatsäohlich handelt es sich hier auch um sehr bedeutende Kraftquellen, denen gegenüber unsere Wasserkrälte an Umfang stark in den Hintergrund treten.

Die Berechnung des Windrades geschieht heute allgemein in der Weise, daß man von den Erfahrungswerten über den Winddruck auf gerade oder gewölbte Flächen ausgeht, die schrăg zum Wind gestellt sind ${ }^{3}$ ). Bei dieser Berechnungsweise tritt der EinfluB von Form und Krümmung der Schaufel nicht unmittelbar in die Erscheinung. Es kommt hierbei nicht zum Ausdruck, daB die Arbeitsleistung des Windrades ausschlieblich aui der Ablenkung des Windes durch die Schaufelflächen berubt, also die Verbältnisse sehr ahnliche sind wie bei den Turbinen. Einige der in neuester Zeit erschienenen Arbeiten ${ }^{*}$ ) benützen die für die Erkenntnis der Wirkung der Luftschraube geschaffene Theorie der idealen Strahlbewegung; diese Theorie nimmt die Schaufelfiäche als Schraubenfläche an und gibt keinen Aufscbluß über die $\mathrm{K}_{\mathrm{u}}$ ümmung der Schaufelprofile, die nach der Eriahrung einen zu beachtenden Einfluß besitzt. Die BehandInng des Windrades als Umkehrung des Propellers ist nun zweifellos fïr die Erlangung eines allgemeinen Einblicks zweckmäßig und wertroll. Sie ist aber nur in beschränktem Maßo zulässig, wie bei-

1) S. z. B. A. u. L. Føppl, Drang und Zrang, 1. Bd, Mtinchen 1920, 8. 306.

) Leon und Zidljeky, Die Ausntitzung des 'Materials in gelochten Korpern. Zeitsehr. Ver. delitsch Ing. 915, s. 11 .

3, Otto Stertz, Moderne Windturbinen, Leipaig 1912. Vergl. alleh Paul La Cour, Die Windkraftmaschinen und thre Anwendung, deutsch von Dr. Joh. Kaufmann, Lelpzig 1905.

1) M. Munk, Ueber rom WInde getriebene Luftschrauben, Zettsehrift fur Flugtechnik und Motorluftschiffahrt 1920, R. 220, ferner Wilh. Hoff, Theorig der idealen Windkraftmasehinen, ebenda, S. 223, endilch A. Betz, Das Maximum der theoretiseh moglichen Ausnutzung des windes durch Windmotore, Zeltschrift für das gesamte Turbjnenwesen 1920, s. 307. 